\title{
A Characterization of Consistent Marked Graphs
}

FACULTY OF APPLIED MATHEMAT/CS

UNIVERSITY OF TWENTE

ENSCHEDE, THE NETHERLANDS

\begin{abstract}
A marked graph is obtained from a graph by giving each point either a positive or a negative sign. Beineke and Harary raised the problem of characterizing consistent marked graphs in which the product of the signs of the points is positive for every cycle. In this paper a characterization is given in terms of fundamental cycles of a cycle basis.
\end{abstract}

\section{INTRODUCTION}

The background of the problem is the modeling of relations between persons in psychology. Cartwright and Harary [3] considered graphs in which the points represent the persons and the lines represent relations that are given positive and negative signs representing the nature of the relationship. Such a graph $S$ is called signed, and is called balanced if the product of the signs of the lines, taken as numbers +1 and -1 , is positive for every cycle in $S$. The characterization of balanced signed graphs is well known and due to Harary [4].

Theorem 1. A signed graph is balanced if and only if there is a partition of its point set into two subsets (one possibly empty) such that every positive line joins two points in the same subset and every negative line joins two points from different subsets.

Harary and Kabell [5] developed a simple algorithm to detect balance in signed graphs. Beineke and Harary [2] investigated marked graphs, the analogue of signed graphs, in which the points are given signs. A marked graph $M$ is consistent if and only if the product of the signs of the points is positive for every cycle in $M$. We shall speak of positive and negative points and cycles, for short.

Journal of Graph Theory, Vol. 16, No. 1, 17-23 (1992)

(C) 1992 John Wiley \& Sons, Inc.

CCC 0364-9024/92/010017-07\$04.00 
Let $T$ be an arbitrary spanning tree of a marked graph $M$. As usual, the lines of $M$ that are not in $T$ will be called chords of $T$, while the lines in $T$ will be called branches. Each chord $e_{i}$ determines a cycle $C_{i}$, called a fundamental cycle. The number of these fundamental cycles is $m(M)$ and is called the cycle rank of $M$. The set of lines of a cycle $C_{i}$ will also be denoted by $C_{i}$, and $C_{i} \oplus C_{j}$ will denote the symmetric difference of the line sets of the cycles $C_{i}$ and $C_{j}$ as well as the graph induced by this set of lines. Each of the $m(M)$ chords determines a fundamental cycle, and we recall the fact that every cycle is the sum (symmetric difference) of the fundamental cycles determined by the chords contained in the cycle.

\section{MAIN THEOREM}

The characterization centers on the markings of the end points of paths that are common to pairs of fundamental cycles. Two fundamental cycles clearly consist of two different chords and two paths belonging to $T$. Their intersection is therefore empty, one point, or a path of positive length, which we call their common path.

The following examples should clarify the nature of the characterization.

In each of the marked graphs of Figure 1, the markings have been indicated, as has a spanning tree $T$ (by thick lines). In both cases the two fundamental cycles are consistent because they contain an even number of negative points. However, in $M_{1}$ the cycle of length 5 , which is the sum of the two fundamental cycles, is negative, whereas in $M_{2}$ it is positive. The two fundamental cycles share a line, which has one positive and one negative point in $M_{1}$, but two negative points in $M_{2}$. This feature turns out to be the essential difference that makes the second graph, but not the first, consistent.

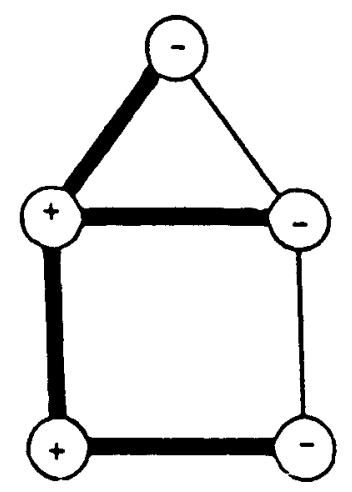

$M_{1}$

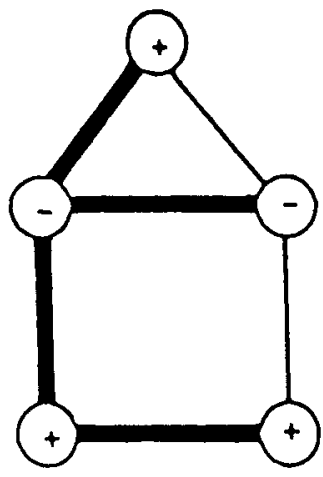

$\mathrm{M}_{2}$

FIGURE 1 
Theorem 2. A marked graph $M$ is consistent if and only if, for any spanning tree $T$, all fundamental cycles are positive and all common paths of pairs of fundamental cycles have end points with the same marking.

Proof. First, assume that $M$ is consistent and let $T$ be a spanning tree of $M$. By definition all fundamental cycles must be positive. Let $C_{1}$ and $C_{2}$ be a pair of fundamental cycles with a path, say from $v$ to $w$, in common. $M$ then contains three disjoint paths from $v$ to $w$. Suppose $v$ and $w$ have opposite markings. Consider the respective numbers of negative points on these three paths, excluding $v$ and $w$. As all cycles are positive, any two of these three numbers have to differ in parity, which is impossible. It follows that $v$ and $w$ have the same marking. (This argument can already be found in the paper of Beineke and Harary).

We now assume that the conditions on the fundamental cycles hold and show that every cycle is positive, using induction on the number of chords. This then proves that $M$ is consistent.

If a cycle contains only one chord, it is a fundamental cycle and is therefore positive. If a cycle $C$ contains two chords $e_{1}$ and $e_{2}$, corresponding say to fundamental cycles $C_{1}$ and $C_{2}$, then $C=C_{1} \oplus C_{2}$ also contains two paths $P_{1}$ and $P_{2}$ in $T$. Let these two paths be connected in $T$ by a path $P$ with end points $v$ and $w$, the common path of the cycles $C_{1}$ and $C_{2}$. Note that $v$ and $w$ must be distinct, since otherwise $C_{1} \oplus C_{2}$ consists of two cycles instead of one. By hypothesis $v$ and $w$ have the same marking. If the number of negative points on $P$ is even, then the number of negative points on $C_{1}$, excluding those of $P$, must be even too. Similarly, the number of negative points on $C_{2}$, excluding those of $P$, must be even. $C$ then has an even number of negative points. If the number of negative points on $P$ is odd, the same result can be derived in an analogous way. This proves that $C$ is positive. The argument used is very similar to that used to prove the necessity of the conditions before.

The induction step uses the same line of thought. Assume that every cycle containing $p$ or fewer chords of $T$ is positive, and let $C$ be a cycle with $p+1$ chords $e_{1}, e_{2}, \ldots, e_{p+1}$. Let $C_{i}$ be the fundamental cycle for chord $e_{i}$, and let $C^{*}=C_{1} \oplus C_{2} \oplus \cdots \oplus C_{p}$. Then $C^{*}$ is a collection of line-disjoint cycles $B_{1}, B_{2}, \ldots, B_{q}$, each of which must be positive by the induction hypothesis. Let $s^{*}$ be the total number of occurrences of negative points in the $B_{j}$, and let $s_{p+1}$ and $s$ denote the number of negative points on $C_{p+1}$ and $C$, respectively. Both $s^{*}$ and $s_{p+1}$ are even. We will show that $s$ must also be even, from which the result will follow.

Note that $C^{*}=C_{p+1} \oplus C$, so any point is on none, two, or four lines in $C^{*}$. We consider now the negative points in $C \cup C_{p+1}$, distinguishing several cases.

Case (i). Those points on four lines of $C^{*}$. Each such point $v$ contributes 2 to $s^{*}$, and 1 each to $s_{p+1}$ and $s$, so the parities of its contribution to $s$ and $s_{p+1}+s^{*}$ are the same. 
Case (ii). Those points $v$ on exactly two of the three entries $C, C^{*}$, and $C_{p+1}$. Each contributes exactly 1 to two of the three quantities $s, s^{*}$, and $s_{p+1}$, and so its contributions to $s$ and $s_{p+1}+s^{*}$ have the same parity.

Case (iii). Those points $v$ on $C, C_{p+1}$, and on a cycle $B_{j}$. Such a point contributes 1 to $s$ and 2 to $s_{p+1}+s^{*}$. The remainder of the proof consists in showing that there are an even number of such negative points, and so the parity of their contribution to both $s$ and $s_{p+1}+s^{*}$ is even. We will do this by showing that these points occur in pairs.

The fundamental cycle $C_{p+1}$ consists of a chord $e_{p+1}$ and a path $P$ in $T$. The path $P$ can be split into subpaths according to the occurrence of its lines in $C^{*}$ or not. Let $c_{1}, c_{2}, \ldots, c_{k}$ be the dividing points and let $P_{1}, P_{2}, \ldots, P_{k}$ be the corresponding paths that are alternately in $C^{*}$ and not in $C^{*}$. We consider these points together with the end points $c_{0}$ and $c_{k+1}$ of $e_{p+1}$. Each subpath of $P$ that belongs to $C^{*}$ determines a pair of points, its end points. The negative points amongst these end points are precisely the points considered in Case (iii). We will show that the end points of $P_{i}$ have the same marking. We assume that these end points are $c_{i-1}$ and $c_{i}$.

Let $d_{1}, d_{2}, \ldots, d_{l}$ be points, different from $c_{i-1}$ and $c_{i}$, at which fundamental cycles that constitute $C$ meet $C_{p+1}$. In case only one fundamental cycle has a path in common with $C_{p+1}$, these points are absent, and $c_{i-1}$ and $c_{i}$ have the same marking as they are the end points of the common path $P_{i}$ of the two fundamental cycles.

Consider the subpaths of $P_{i}$ that are common paths of $C_{p+1}$ and fundamental cycles constituting $C$. These paths have the following properties. They cover every line of $P_{i}$ an odd number of times. Their end points have the same marking. Every point $d$ is an end point of an even number of these paths. The $c_{i-1}$ and $c_{i}$ are end points of an odd number of these paths.

We now use induction on the number of such covering paths. Let there be $m+1$ paths covering $P_{i}$ in the described way. If every line is covered exactly once, the paths are consecutive and the result follows. Therefore let at least one line of $P_{i}$ be covered three times or more. We distinguish three cases.

Case (1). Two covering paths, from $d^{\prime}$ to $d$ respectively from $d$ to $d^{\prime \prime}$, have only an end point $d$ in common. These two consecutive paths form a path with end points $d^{\prime}$ and $d^{\prime \prime}$ with the same marking. Replacing the two paths by this path, we obtain a collection of $m$ paths covering $P_{i}$ in the described way. Note that the number of paths with end point $d$ has been lowered by 2 . By induction hypothesis the end points of $P_{i}$ have the same marking.

Case (2). Two covering paths from $d$ to $d^{\prime}$ (or two from $d$ to $d^{\prime \prime}$ ) have the path from $d$ to $d^{\prime}$ in common. The $d^{\prime}$ and $d^{\prime \prime}$ again have the same mark- 
ing. The lines of the path from $d$ to $d^{\prime}$ are covered at least three times. We now replace these two paths by the path from $d^{\prime}$ to $d^{\prime \prime}$ and obtain a collection of $m$ covering paths. The lines that belonged to the path from $d$ to $d^{\prime}$ are now covered two times less, i.e., at least one time and still an odd number of times. The collection of $m$ paths covers $P_{i}$ in the described way, and by induction hypothesis the end points of $P_{i}$ have the same marking.

Case (3). Two covering paths have the same end points $d^{\prime}$ and $d^{\prime \prime}$. In this case both paths are omitted from the collection of covering paths and we obtain a collection of $m-1$ paths that cover $P_{i}$ with the same properties.

This proves that the negative points mentioned before in Case (iii) occur in pairs and the proof has been completed.

\section{EXAMPLE}

In Figure 2a, a typical path $P_{i}$ is depicted as it is constituted by common paths that have end points at the points $c_{i-1}, d_{1}, \ldots, d_{7}, c_{i}$.

All lines are covered once or thrice. Two fundamental cycles have common paths with $C_{p+1}$ with end points $d_{1}$ and $d_{2}$. Two common paths between $d_{5}$ and $d_{6}$ and between $d_{6}$ and $d_{7}$ form a path between $d_{5}$ and $d_{7}$ with end points with the same marking. Two common paths between $d_{3}$ and $d_{4}$ and between $d_{3}$ and $d_{7}$ overlap between $d_{3}$ and $d_{4}$. The path between $d_{4}$ and $d_{7}$ has end points with the same marking. The covering of the lines can be thinned out to that of Figure $2 \mathrm{~b}$. Repetition of the procedure, by removing overlapping parts of the paths between $d_{5}$ and $d_{7}$ and between $d_{5}$ and $c_{i}$, leads to a covering of the lines by exactly one subpath of $P_{i}$, while $c_{i-1}$ and $d_{4}, d_{4}$ and $d_{7}$ and $d_{7}$ and $c_{i}$, and therefore $c_{i-1}$ and $c_{i}$ have the same marking.

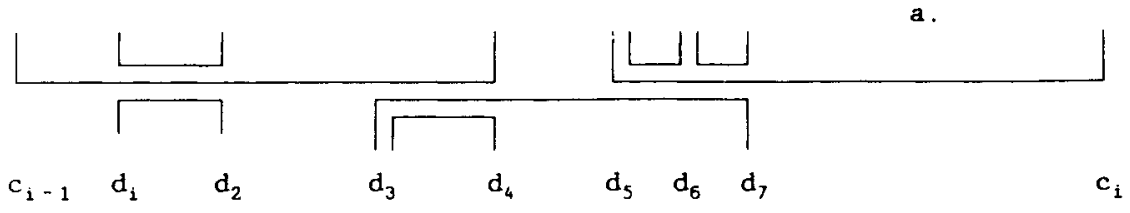

b.

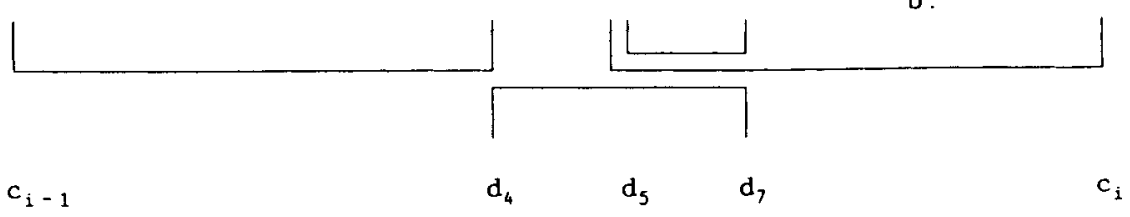


The characterization given in Theorem 2 implies a polynomial algorithm for determining whether a marked graph is consistent. The number of fundamental cycles is $O\left(n^{2}\right)$, where $n$ is the order of $M$. These have to be checked for positivity. The number of their common paths is $O\left(n^{4}\right)$ and these must be checked on equality of the markings of end points.

\section{DISCUSSION}

The nature of the characterization is completely different from that searched for by Beineke and Harary, who reduced the investigation of consistent marked graphs to that of tripartite graphs, with two classes of negative points and one class of positive points. The author first followed that line of thought, with the following results.

By subdividing each line between two negative points by one positive point, any tripartite graph $T$ like that considered above can be turned into a bipartite graph $B$ that is consistently marked if and only if $T$ is consistently marked. This simple transformation reduces the problem to characterizing consistent bipartite marked graphs with one class of positive and one class of negative points. One can prove quite easily that these graphs are consistent if and only if all cycles have lengths that are multiples of 4 , therefore containing an even number of negative points.

This characterization does not lead to a polynomial algorithm, as the number of cycles to be checked may be exponential. However, a transformation of a signed graph $S$ into a marked graph $M(S)$ can be given that has an interesting consequence. Let $S$ be a signed graph. Its points are given a positive marking. Each line with a negative sign is subdivided by a point that is given a negative marking. Thus a marked graph $M(S)$ is obtained. Again it is easy to see that $S$ is balanced if and only if $M(S)$ is consistent. The interesting consequence is that the characterization of consistent marked graphs in terms of cycles applied to a bipartite graph associated with $M(S)$ can be used to derive Theorem 1 as a corollary.

It was pointed out by one referee that the problem had already been solved by Rao [6] and that work related to Rao's paper had been published by Acharya [1]. The paper by Acharya contains a characterization of a class of consistent marked graphs only, namely those marked graphs in which the subgraph induced by the negative points is connected. In Rao's paper, a full characterization is indeed given. However, there is a problem with this characterization. Rao follows the line of investigation that the author first followed too. More and more necessary conditions are derived, starting with the obvious demand that the graph induced by the negative points should be bipartite. The resulting complex set of conditions is then shown to be sufficient. As a result of this approach the full statement of the characterization would take about two full pages. The paper was reviewed in Mathematical Reviews by L.W. Beineke, who refrained from giving the full 
description of the result, probably for this reason. The algorithm that Rao describes for checking consistency contains 13 steps in which certain structures are to be found by a variety of (polynomial) algorithms. In contrast to this, our characterization seems much simpler and easier to check.

\section{ACKNOWLEDGMENT}

The authors got involved with the problem of characterizing consistent marked graphs during a stay in Ann Arbor in the academic year of 19801981. Helpful remarks from Frank Harary on first results derived then are gratefully acknowledged. The author also thanks the referee for criticism that led to improvement of the presentation.

\section{References}

[1] B. D. Acharya, Some further properties of consistent marked graphs. Indian J. Pure Appl. Math. 15 (1989) 837-842.

[2] L.W. Beineke and F. Harary, Consistent graphs with signed points Riv. di Mat. per le Sci. Econ. e Soc. 1 (1978) 81-88.

[3] D. Cartwright and F. Harary, Structural balance: A generalization of Heider's Theory. Psych. Rev. (1956) 277-293.

[4] F. Harary, On the notion of balance of a signed graph. Mich. Math. J. 1 (1953) 143-146.

[5] F. Harary and J. A. Kabell, A simple algorithm to detect balance in signed graphs. Math. Soc. Sci. 1 (1980) 131-136.

[6] S. B. Rao, Characterization of harmonious marked graphs and consistent nets. J. Combinat. Inform. Syst. Sci. 9 (1984) 97-112. 